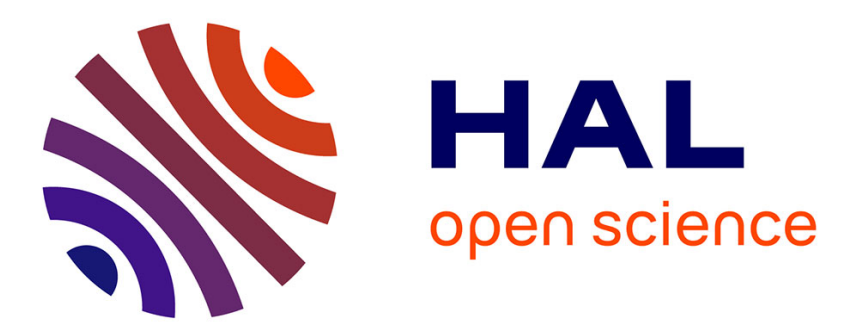

\title{
Auto-adaptive and Dynamical Clustering for Open-Circuit Fault Diagnosis of Power Inverters
}

Thanh Hung Pham, Sanda Lefteriu, Cécile Labarre, Eric Duviella, Stéphane Lecoeuche

\section{- To cite this version:}

Thanh Hung Pham, Sanda Lefteriu, Cécile Labarre, Eric Duviella, Stéphane Lecoeuche. Auto-adaptive and Dynamical Clustering for Open-Circuit Fault Diagnosis of Power Inverters. ECC 2019 - European Control Conference, Jun 2019, Naples, Italy. hal-02123169v2

\section{HAL Id: hal-02123169 \\ https://hal.science/hal-02123169v2}

Submitted on 24 Nov 2020

HAL is a multi-disciplinary open access archive for the deposit and dissemination of scientific research documents, whether they are published or not. The documents may come from teaching and research institutions in France or abroad, or from public or private research centers.
L'archive ouverte pluridisciplinaire HAL, est destinée au dépôt et à la diffusion de documents scientifiques de niveau recherche, publiés ou non, émanant des établissements d'enseignement et de recherche français ou étrangers, des laboratoires publics ou privés. 


\title{
Auto-adaptive and Dynamical Clustering for Open-Circuit Fault Diagnosis of Power Inverters
}

\author{
Thanh Hung Pham, Sanda Lefteriu, Cécile Labarre, Eric Duviella, Stéphane Lecoeuche
}

\begin{abstract}
This paper presents a fault diagnosis approach for single open-circuit faults in inverters entirely from measurements of the stator currents. These measurements are used to extract the feature data; the feature data is then used to create clusters in an online, adaptive and unsupervised way. Auto-adaptive and Dynamical Clustering (AUDyC) is the algorithm employed for this step. Based on the derived clusters, appropriate formulations for the data labelling and fault detection and isolation are proposed. The effectiveness of the approach is validated on simulation and experimental data.
\end{abstract}

\section{INTRODUCTION}

An inverter is a power converter that changes Direct Current (DC) to Alternative Current (AC). It is usually used to connect DC sources (e.g., battery) and AC electrical devices (e.g., AC electric motor). Fault diagnosis of the 3-phase inverter associated to a 3-phase motor represents an active area of research [1]. Because of aging or abnormal operating conditions, semiconductor switches, such as Insulated-Gate Bipolar Transistors (IGBTs), are the most vulnerable components in the inverter. Their failure is due to the Short-Circuit (SC) and Open-Circuit (OC) faults. The SC fault leads to a high current which is destructive and makes the system shut down immediately thanks to standard protection systems [2]. However, when the OC fault happens, the system can still run with degraded performance causing secondary faults on other system components. Thus, this paper focuses on OC fault diagnosis of 3-phase power inverters.

In terms of methodology, most researchers focus on one of the following approaches: model-based, signal-based, datadriven and hybrid [3]. The model-based approach uses the system model, obtained by physical principles or system identification techniques, to predict the output, which is compared to the measured output for diagnosing the fault [4]. It should be noted that this model is generally difficult to derive for a complex system, e.g., an asynchronous motor associated with an inverter. Instead of system models, the signal-based approach considers the measured signals for extracting features which are used, together with prior knowledge on the symptoms of healthy systems, to make diagnosis decisions [1], [5]. However, this approach does not take into consideration the dynamics of the input signal which is usually impacted by unknown disturbances or unbalanced

Thanh Hung Pham, Sanda Lefteriu, Cécile Labarre, Eric Duviella, Stéphane Lecoeuche are with Institut Mines Telecom Lille Douai, IA, F-59508 Douai, France \{thanh-hung.pham, sanda.lefteriu, cecile.labarre,

eric.duviella, stephane.lecoeuche\}

eimt-lille-douai.fr conditions. In contrast to the two previous approaches, datadriven approaches consider a large volume of data and prior knowledge of considered systems to make fault diagnosis decisions thanks to data classification or clustering. Nevertheless, this approach suffers from a high computational cost, and may not identify unknown fault types. To combine the strengths of the previous approaches, our paper proposes a hybrid approach where signal-based and data-driven methods are combined.

For the 3-phase inverter, such a hybrid approach may be based on measurements of voltages and/or currents. Voltagebased methods use measurements of the three voltages at the inverter output [2]. This method is independent of the load, but requires additional sensors. The latter disadvantage motivates us to develop a current-based method, the load independence being ensured by simply normalizing the current data [1], [6].

The data-driven approach is used to determine the feature classes, which are feature vector sets characterizing system operation modes. In the context of OC fault diagnosis for inverters, previous research proposed well-established statistical methods such as Principle Component Analysis (PCA) [2] and Support Vector Machines (SVM) [7], [8]. For these previous methods, reference classes are already available from the training data, and one needs to determine to which class the new feature vector belongs to. For this reason, they are called supervised methods, and unknown operation modes may not be detected during the actual system operation. To avoid this, Neural Networks (NN) can be applied to learn and diagnose the OC faults of inverters thanks to their powerful ability in nonlinear approximation and adaptive learning [9]-[12]. However, in these papers, the number of classes is fixed, while the characteristics of the class is adapted to include the new feature vector. This issue may be tackled using adaptive unsupervised methods, where reference classes (called clusters) are not available a priori, but are created online. To the author's best knowledge, such a method has yet to be applied to diagnose the OC fault of inverters. Moreover, most of existing clustering methods do not predict the time evolution of modelled data classes and their fusion.

In this paper, we employ the normalized DC current for the feature extraction, and Auto-adaptive and Dynamical Clustering (AUDyC) algorithm [13], [14] for feature clustering. AUDyC does online, adaptive and unsupervised feature learning, hence the unknown modes of operation can be learnt from the measurement data. The main contribution of our work resides in the application of AUDyC to perform 
single OC fault diagnosis of inverters by identifying representative data classes. Furthermore, appropriate conditions for labelling classes and for diagnosing faults are presented.

The paper is organized as follows. Section II formulates the fault diagnosis problem. Section III describes the proposed method, and section IV validates it on two test cases: the first considers simulation data from a 3-phase inverter, while the second is measurement data from a 5phase inverter. Section $\mathrm{V}$ concludes the paper and presents future work directions.

\section{THE INVERTER IN NORMAL AND FAULTY OPERATION MODES}

This section presents the 3-phase inverter associated with a motor in normal and faulty operation modes.

\section{A. Inverter operation}

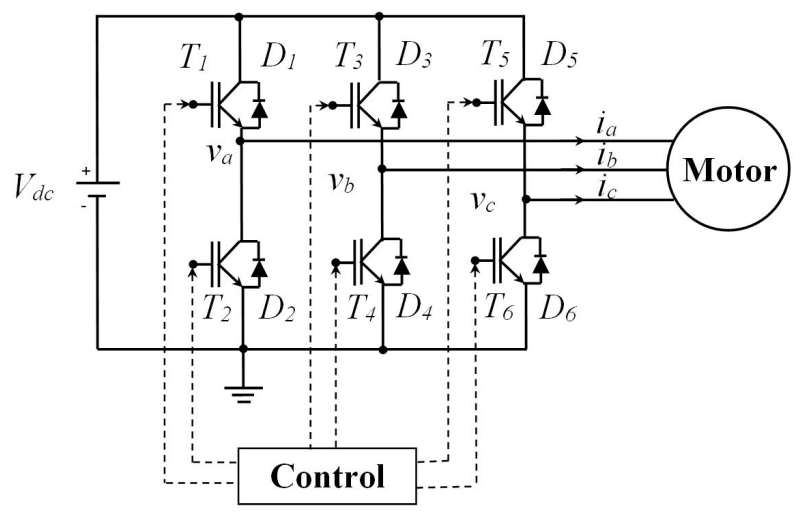

Fig. 1: 3-phase inverter driving a motor [2].

The considered system is a combination of a 3-phase inverter and a motor with the following specifications: star coupling for the stator with the isolated neutral, symmetric motor electrical circuit operating under open-loop control based on pulse width modulation (PWM). We assume that no fault occurs during the motor starting period. Each inverter leg is composed of two IGBTs and two inverse diodes as illustrated in Fig. 1.

TABLE I: Operation modes, diode states, transistor states and electric voltage of leg $a$.

\begin{tabular}{|l|l|l|l|l|}
\hline \multirow{5}{*}{$T_{2}$ open } & \multicolumn{2}{|c|}{$i_{a}<0$} & \multicolumn{2}{c|}{$i_{a}>0$} \\
\cline { 2 - 5 } & $T_{1}$ open & $T_{1}$ closed & $T_{1}$ open & $T_{1}$ closed \\
\hline \multirow{5}{*}{ OC fault } & normal or & OC fault or & OC fault & $\begin{array}{l}\text { normal or } \\
\text { OC fault or } \\
\text { SC fault }\end{array}$ \\
\hline \multirow{5}{*}{$T_{2}$ closed fault } & & SC & SC \\
& $D_{1}$ closed & $D_{1}$ closed & $D_{1}$ open & $D_{1}$ open \\
& $D_{2}$ open & $D_{2}$ open & $D_{2}$ closed & $D_{2}$ open \\
\cline { 2 - 5 } & $v_{a}=V_{d c}$ & $v_{a}=V_{d c}$ & $v_{a}=0$ & $v_{a}=V_{d c}$ \\
\hline \multirow{5}{*}{} & normal or & & normal or & \\
& OC fault or & & OC fault or & \\
& SC fault & SC fault & SC fault & SC fault \\
\cline { 2 - 5 } & $D_{1}$ open & & $D_{1}$ open & \\
& $D_{2}$ open & & $D_{2}$ closed & \\
\cline { 2 - 5 } & $v_{a}=0$ & & $v_{a}=0$ & \\
\hline
\end{tabular}

Since the three legs of the inverter are symmetric, only the operation states of leg $a$ are described in Table I. An inverter operation mode is defined as a time sequence of IGBT operation states. In the normal operation mode, $T_{1}$ and $T_{2}$ are alternatively open or closed with the PWM switching frequency. Note that the dead time is not considered here, i.e., it is assumed to be zero. When IGBT $T_{1}$ or $T_{2}$ is closed all the time, the inverter is in SC fault mode, which is not considered in this work. When IGBT $T_{1}$ or $T_{2}$ is open all the time, the inverter is in OC fault mode, which will be investigated in the next subsection.

\section{B. Open-circuit fault analysis}

Fig. 2 illustrates the profiles of the three stator AC currents in steady-state where the system is in the normal mode before $0.40 \mathrm{~s}$, and in the $T_{1}$ OC fault mode after $0.40 \mathrm{~s}$.

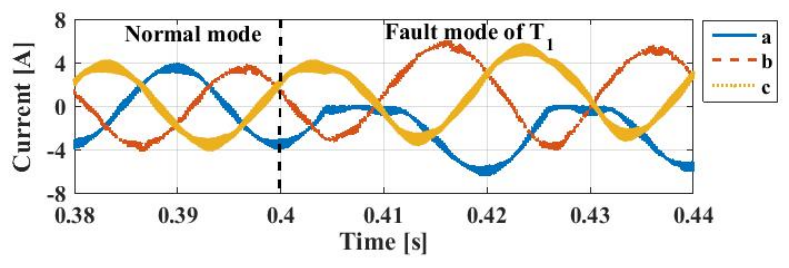

Fig. 2: Phase AC currents in the normal and $T_{1}$ faulty modes.

The switching frequency of IGBTs is assumed to be much higher than the sinusoidal signal frequency. In the normal operation mode, when we generate the PWM signal to control the IGBTs based on three sinusoidal signals with frequency $f$ and shifted by $2 \pi / 3$ between their phases, the three profiles of the stator currents are also sinusoidal with the same frequency and phase shifts at the steady state. Indeed, there is still the small oscillation in the current profiles because of IGBT switching (see Fig. 2).

The inverter is in $T_{1}$ (respectively $T_{2}$ ) OC fault mode if $T_{1}$ (respectively $T_{2}$ ) is open all the time while other IGBTs are still alternatively closed and open. In this case, if the current $i_{a}(t)$ is negative, the current profiles are still sinusoidal. It can be seen in Fig. 2 where the fault happens at $0.4 \mathrm{~s}$. This is due to the fact that the electric potential profile of phase a, $v_{a}(t)$, does not change with respect to the normal mode according to Table I. However, if the current $i_{a}(t)$ is positive, the electric potential of phase a, $v_{a}(t)$, is always equal to zero, and the phase-a current, $i_{a}(t)$, reduces to zero quickly and stays like this for about half a period [7].

Thus, we can deduce an important characteristic of the stator currents in the faulty mode: the positive part of the phase-a current, $i_{a}(t)$, is "cut-off" when $T_{1}$ is faulty. Consequently, only the phase-a current is always negative during the $T_{1}$ fault mode. This results in the fact that the ratio between its average and the average of its absolute value on a fundamental signal period is equal to -1 . Moreover, these ratios for the other phase currents are theoretically constant. Similarly, this observation can be easily extended to the faulty cases of the other IGBTs. Hence, each mode can be represented by a vector constructed from these ratios. These presented fault characteristics will be exploited to formulate the feature variable in the next section. 


\section{HYBRID FAULT DIAGNOSIS METHOD}

In this section, a hybrid diagnosis method is presented which includes the following steps (as illustrated in Fig. 3): feature extraction, data clustering, labelling and fault detection and isolation.

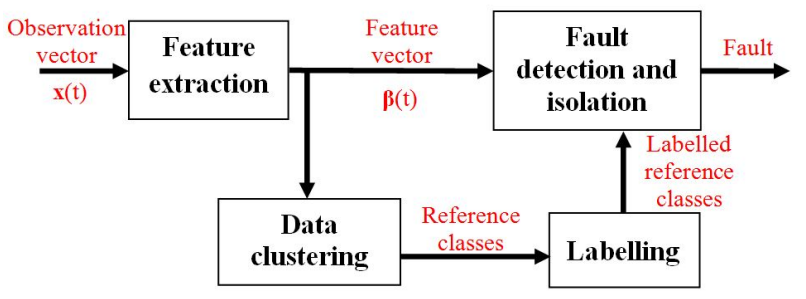

Fig. 3: Fault diagnosis algorithm.

\section{A. Feature extraction}

In this step, we focus on finding the feature vector which gathers all the feature variables. The linear space representing this vector is called feature space where feature vectors corresponding to different operation modes are located in different regions in this space.

Let $\mathbf{x}(t), \boldsymbol{\beta}(t)$ be, respectively, the observation and feature vectors at time instant $t=k t_{s}$, where $t_{s}$ is the sampling time, and $k \in \mathbb{N}$ is the sampling time index. In our case, the measured variables are the 3-phase currents denoted by $i_{a}(t), \quad i_{b}(t), \quad i_{c}(t) \in \mathbb{R}$. Therefore, the observation vector is defined as $\mathbf{x}(t)=\left[\begin{array}{lll}i_{a}(t) & i_{b}(t) & i_{c}(t)\end{array}\right]^{T} \in \mathbb{R}^{3}$. According to the faulty mode analysis in Section II, the feature vector is chosen as:

$$
\begin{aligned}
& \boldsymbol{\beta}(t)=\left[\begin{array}{lll}
\beta_{a}(t) & \beta_{b}(t) & \beta_{c}(t)
\end{array}\right]^{T} \in \mathbb{R}^{3}, \text { with } \\
& \beta_{l}(t)=\frac{\left\langle i_{l}(t)\right\rangle}{\left\langle i_{l}(t) \mid\right\rangle}, l=a, b, c,
\end{aligned}
$$

where $\langle f(t)\rangle=\sum_{k=0}^{N-1} f\left(t-k t_{s}\right) / N$ is the average of function $f(t), N \in \mathbb{N}$ is the number of time samples on a fundamental signal period. Notice that $-1 \leq \beta_{l}(t) \leq 1$. When the lower transistor of phase $l$ is faulty, $i_{l}(t)>0$ $\forall t$, and thus, $\beta_{l}(t)=1$. Similarly, when the upper transistor of phase $l$ is faulty, $\beta_{l}(t)=-1$. These remarks illustrate the relevance of (1b) for the feature extraction. The feature vector will be used as the input to the following clustering procedure.

\section{B. Data clustering}

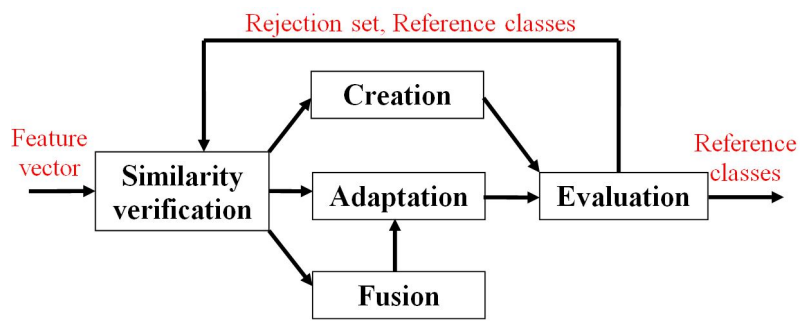

Fig. 4: AUDyC algorithm [14].
Here, we describe AUDyC, the algorithm chosen for data clustering, thanks to its online, adaptive and unsupervised learning capabilities [13], [14]. AUDyC automatically creates data classes in the feature space from the feature data. In our case, these classes represent the normal and single OC fault (i.e., a single transistor is faulty at a time) modes. Since each operation mode is ideally represented by a unique nominal feature vector, we consider a simplified formulation of AUDyC in this paper. This simplification is obtained by assuming that the data of each class follows a gaussian distribution around the nominal feature vector. Thus, a class is characterized by the mean vector, the covariance matrix and a set of feature vectors. Class parameters are determined in the following 5 main procedures: the similarity verification, the creation, the adaptation, the fusion and the evaluation of classes, as illustrated in Fig. 4. At each time instant, a loop over these steps is performed on a vector set composed of the new feature vector and the rejected vectors from the previous loop. After each iteration, a vector of this set is clustered, and class parameters are modified.

Similarity verification: This procedure finds the class to which the new feature vector, $\boldsymbol{\beta}(t)$, belongs, by quantifying similarities between this vector and existing classes based on membership functions defined as:

$$
\mu_{j}(\boldsymbol{\beta}(t))=\exp \left(-\frac{1}{2} \tilde{\boldsymbol{\beta}}(t)^{T} \Sigma_{j}^{-1}\left(t-t_{s}\right) \tilde{\boldsymbol{\beta}}(t)\right),
$$

where $\overline{\boldsymbol{\beta}}_{j}(t)$ and $\Sigma_{j}(t)$ are, respectively, the mean vector and the covariance matrix of class $\mathbb{C}_{j}$, and $\tilde{\boldsymbol{\beta}}(t)=\boldsymbol{\beta}(t)-\overline{\boldsymbol{\beta}}_{j}(t-$ $\left.t_{s}\right)$. Then, all classes $j$ satisfying $\mu_{j}(\boldsymbol{\beta}(t)) \geq \mu_{\text {min }}$ with the given threshold $\mu_{\text {min }}$ are gathered in a set denoted by $\mathbb{C}_{w i n}$. Based on the cardinality of this set, we determine the next steps.

Creation: If card $\left(\mathbb{C}_{\text {win }}\right)=0$, a new class $\mathbb{C}_{j}$ is created with the following parameters:

$$
\left\{\begin{array}{l}
\overline{\boldsymbol{\beta}}_{j}(t)=\boldsymbol{\beta}(t) \\
\Sigma_{j}(t)=\sigma^{2} \mathbf{I}_{3}
\end{array}\right.
$$

where $\mathbf{I}_{3}$ is the identity matrix of size 3 , and $\sigma \in \mathbb{R}^{+}$is a chosen parameter which determines the learning capacity of the created class at its first moment.

Adaptation: If card $\left(\mathbb{C}_{\text {win }}\right)=1$, the similarity class $\mathbb{C}_{j} \in$ $\mathbb{C}_{\text {win }}$ is adapted. Let $n_{j}(t) \in \mathbb{N}$ be the number of feature vectors belonging to $\mathbb{C}_{j}$ at time instant $t$. If $n_{j}\left(t-t_{s}\right)<$ $N_{\text {min }}$, where $N_{\text {min }}$ is a given threshold, the feature vector is stored in the memory of class $\mathbb{C}_{j}$. Moreover, the parameters of $\mathbb{C}_{j}$ are modified using the new feature vector:

$$
\left\{\begin{aligned}
n_{j}(t) & =n_{j}\left(t-t_{s}\right)+1, \\
\overline{\boldsymbol{\beta}}_{j}(t) & =\overline{\boldsymbol{\beta}}_{j}\left(t-t_{s}\right)+\frac{\tilde{\boldsymbol{\beta}}(t)}{n_{j}(t)}, \\
\Sigma_{j}(t) & =\Sigma_{j}\left(t-t_{s}\right) .
\end{aligned}\right.
$$

If $N_{\min } \leq n_{j}\left(t-t_{s}\right)<N_{\max }$, where $N_{\max }$ is a given threshold, the feature vector is stored. $n_{j}(t)$ and $\overline{\boldsymbol{\beta}}_{j}(t)$ are modified according to the same formulas in (4), and $\Sigma_{j}(t)$ 
is given as:

$$
\Sigma_{j}(t)=\frac{n_{j}(t)-2}{n_{j}(t)-1} \Sigma_{j}\left(t-t_{s}\right)+\frac{\tilde{\boldsymbol{\beta}}(t) \tilde{\boldsymbol{\beta}}(t)^{T}}{n_{j}(t)} .
$$

If $n_{j}\left(t-t_{s}\right)=N_{\max }$, the new feature vector is stored, and the oldest one is forgotten. Therefore, the class parameters are adapted by the following rules:

$$
\left\{\begin{array}{l}
n_{j}(t)=N_{\max }, \\
\overline{\boldsymbol{\beta}}_{j}(t)=\overline{\boldsymbol{\beta}}_{j}\left(t-t_{s}\right)+\frac{\Delta \boldsymbol{\beta}(t)}{N_{\max }}\left[\begin{array}{r}
1 \\
-1
\end{array}\right], \\
\Sigma_{j}(t)=\Sigma_{j}\left(t-t_{s}\right)+\Delta \boldsymbol{\beta}(t) \mathbf{B} \Delta \boldsymbol{\beta}(t)^{T},
\end{array}\right.
$$

with

$$
\left\{\begin{array}{r}
\Delta \boldsymbol{\beta}(t)=\left[\begin{array}{r}
\boldsymbol{\beta}(t)-\overline{\boldsymbol{\beta}}_{j}\left(t-t_{s}\right) \\
\boldsymbol{\beta}\left(t-N_{\max } t_{s}+t_{s}\right)-\overline{\boldsymbol{\beta}}_{j}\left(t-t_{s}\right)
\end{array}\right]^{T}, \\
\mathbf{B}=\frac{1}{N_{\max }}\left[\begin{array}{cr}
1 & \frac{1}{N_{\max }-1} \\
\frac{1}{N_{\max }-1} & -\frac{N_{\max }+1}{N_{\max }-1}
\end{array}\right] .
\end{array}\right.
$$

Fusion: When card $\left(\mathbb{C}_{\text {win }}\right) \geq 2$, if each two classes $\mathbb{C}_{j}, \mathbb{C}_{m} \in \mathbb{C}_{\text {win }}$ satisfy the condition:

$$
\operatorname{tr}\left(\Sigma_{j}(t) \Sigma_{m}^{-1}(t)+\Sigma_{m}(t) \Sigma_{j}^{-1}(t)\right)-6<\epsilon_{f},
$$

all the similarity classes are fused, where $\operatorname{tr}(\mathbf{X})$ is the trace of matrix $\mathbf{X}, \epsilon_{f}$ is a given threshold. The classes are organized in the appearance order. The new class obtained by fusing two first classes is fused with the third class. The procedure is repeated until all the similarity classes are fused. Then, the parameters of the final fusion class is adapted to include the new feature vector. If (8) is not satisfied, only the parameters of the class with the highest value of the membership function in (2) is adapted with the new feature vector.

Evaluation: After every $N_{\min }$ time samples, a noisy cluster $\mathbb{C}_{j}$ is eliminated if $\operatorname{card}\left(\mathbb{C}_{j}\right)<N_{\text {min }} . \mathbb{C}_{j}$ is removed from the set of the reference classes, and all its feature vectors are stored in a rejection set for reuse in the next learning iteration (see Fig. 4).

AUDyC clusters feature data to different classes representing different system operation modes. These classes have no physical meaning, hence, it is necessary to determine their corresponding names in the following labelling procedure.

\section{Labelling}

Based on the physical knowledge available, conditions are formulated to determine the class names. In our case, we assume that there are 7 operation modes: normal mode and 6 single OC fault modes corresponding to the 6 IGBTs in the 3-phase inverter.

Let $\bar{\beta}_{a, j}, \bar{\beta}_{b, j}, \bar{\beta}_{c, j} \in \mathbb{R}$ be, respectively, the coordinates of the mean vector $\overline{\boldsymbol{\beta}}_{j}$ of class $\mathbb{C}_{j}$. From the analysis in the last paragraph of Section II, we remind that the feature variables defined by (1b) are close to zero in the normal mode and converge to 1 or -1 in the faulty modes. Thus, a labelling threshold $\epsilon_{l}$ is given to verify if the mean vectors
TABLE II: Labelling conditions.

\begin{tabular}{|l|r|}
\hline Mode & \multicolumn{1}{|l|}{ Condition } \\
\hline Normal & $\left|\overline{\boldsymbol{\beta}}_{j}\right|<\sqrt{3} \epsilon_{l}$ \\
Fault $T_{1}$ & $-1-\epsilon_{l}<\bar{\beta}_{a, j}<-1+\epsilon_{l}$ \\
Fault $T_{2}$ & $1-\epsilon_{l}<\bar{\beta}_{a, j}<1+\epsilon_{l}$ \\
Fault $T_{3}$ & $-1-\epsilon_{l}<\bar{\beta}_{b, j}<-1+\epsilon_{l}$ \\
Fault $T_{4}$ & $1-\epsilon_{l}<\bar{\beta}_{b, j}<1+\epsilon_{l}$ \\
Fault $T_{5}$ & $-1-\epsilon_{l}<\bar{\beta}_{c, j}<-1+\epsilon_{l}$ \\
Fault $T_{6}$ & $1-\epsilon_{l}<\bar{\beta}_{c, j}<1+\epsilon_{l}$ \\
\hline
\end{tabular}

of the modelled classes are close to these reference values as in Table II. If there is no satisfied condition, the class represents an unknown mode.

Based on these labels, we can determine the mode names of the new feature vectors in the following section.

\section{Fault detection and isolation}

This procedure concentrates on finding fault indicator. Using only the new feature vector for diagnosing the OC fault may cause false alarms due to two reasons: perturbations or temporary passage. The temporary passage implies that the feature vector may rapidly pass through a class which does not represent the actual operation mode. To deal with these false alarm causes, we consider a sequence of consecutive feature vectors, $\mathbb{B}(t)=\left\{\boldsymbol{\beta}\left(t-N_{f} t_{s}+t_{s}\right), \ldots, \boldsymbol{\beta}(t)\right\}$, where $N_{f} \in \mathbb{N}$ is a chosen parameter defining the width of the sequence. We find the class to which most of vectors of $\mathbb{B}(t)$ belong. Then, the number of vectors of $\mathbb{B}(t)$ that belong to this class is used as the fault indicator.

Let $n_{p}(t) \in \mathbb{N}$ be the number of existing classes. We define the indication matrix $\mathbf{M}(t) \in\{0,1\}^{N_{f} \times n_{p}(t)}$ such that:

$$
m_{k j}(t)= \begin{cases}1, & \text { if } \mu_{j}\left(\boldsymbol{\beta}\left(t-k t_{s}+t_{s}\right)\right)= \\ & \max _{p} \mu_{p}\left(\boldsymbol{\beta}\left(t-k t_{s}+t_{s}\right)\right) \geq \mu_{f} \\ 0, & \text { if else }\end{cases}
$$

where $m_{k j}(t)$ is the element on the $k^{t h}$ row and $j^{t h}$ column of matrix $\mathbf{M}(t), \mu_{j}(\boldsymbol{\beta}(t))$ is the membership function of class $\mathbb{C}_{j}$ defined in (2), $\mu_{f}$ is a given indication threshold. The fault indicator is the maximum absolute column sum norm of the matrix $\mathbf{M}(t)$, that is $I(t)=\|\mathbf{M}(t)\|_{1}$.

Let $j_{\max }$ be the index of the column whose absolute column sum is highest. The system operation is in the mode corresponding to class $\mathbb{C}_{j_{\max }}$ if $I(t) \geq I_{f}$, where $I_{f} \in \mathbb{N}$ is a given fault threshold. Otherwise, the system is in an unknown faulty mode. The fault detection strongly depends on the parameters chosen for the AUDyC algorithm. Therefore, the detection time is not easy to be mathematically estimated.

The presented diagnosis procedure is validated using simulation and experimental data in the next section.

\section{Diagnosis Results}

Using the proposed method, this section presents the diagnosis results for the inverter single OC fault based on simulation and experiment data. In Scenario 1, simulation data are obtained using Simscape Electrical toolbox in Matlab. In Scenario 2, experimental data on a 5-phase inverter 
is provided by the L2EP laboratory in Lille, France. The properties of the data and the parameters of the diagnosis algorithm are presented in Table III. In the following result analysis, the mode flag is described by the natural variable fault. It is set to 0 if the operation mode is not detected. Generally, there is rule for choosing the algorithm parameters. Their values given in Table III were obtained by trying different combinations for Scenario 1.

TABLE III: Parameters for the data and the diagnosis algorithm.

\begin{tabular}{|l|l|l|l|}
\hline Parameter & Unit & Scen. 1 & Scen. 2 \\
\hline \multicolumn{3}{|c|}{ Data } \\
\hline Signal frequency & {$[\mathrm{Hz}]$} & 50 & 50 \\
Sample time $t_{s}$ & {$[\mu \mathrm{s}]$} & 80 & 80 \\
PWM switching frequency & {$[\mathrm{Hz}]$} & 5000 & 10000 \\
\hline \multicolumn{3}{|c|}{ AUDyC } \\
\hline Class membership threshold $\mu_{\min }$ & - & 0.7 & 0.7 \\
Initial covariance $\sigma$ & - & 0.8 & 0.8 \\
Minimum class cardinality $N_{\min }$ & - & 150 & 150 \\
Maximum class cardinality $N_{\max }$ & - & 250 & 250 \\
Fusion threshold $\epsilon_{f}$ & - & 3.2 & 3.2 \\
\hline \multicolumn{3}{|c|}{ Labelling } \\
\hline Labelling threshold $\epsilon_{l}$ & - & 0.1 & 0.1 \\
\hline \multicolumn{3}{|c|}{ Fault detection and isolation } \\
\hline Indication threshold $\mu_{f}$ & - & 0.7 & 0.7 \\
Sliding window width $N_{f}$ & - & 150 & 150 \\
Fault threshold $I_{f}$ & - & 130 \\
\hline
\end{tabular}

\section{A. Scenario 1: Simulation data}

The normal mode and 6 alternative single OC fault modes are implemented as described in Table IV. The first row indicates the time durations of each mode.

TABLE IV: Settings and detection times in Scenario 1.

\begin{tabular}{|l|c|c|c|c|c|c|c|}
\hline Time [s] & 0.2 & 0.1 & 0.1 & 0.1 & 0.1 & 0.1 & 0.1 \\
\hline Fault & None & $T_{1}$ & $T_{2}$ & $T_{3}$ & $T_{4}$ & $T_{5}$ & $T_{6}$ \\
\hline fault $_{\text {ref }}$ & 1 & 2 & 3 & 4 & 5 & 6 & 7 \\
\hline Det. time [ms] & & 22.6 & 30.6 & 30.9 & 28.5 & 17.8 & 28.4 \\
\hline
\end{tabular}

The profiles of phase currents, of feature variables and of fault flag are described in Fig. 5. This figure illustrates the remark in Section II.B: when an OC fault happens, the positive (or negative) part of the current in the corresponding leg is cut-off. It results in the -1 (or 1) value of the corresponding feature variable.

Fig. 6 describes the feature classes obtained using AUDyC corresponding to the operation modes. To verify if these classes characterize the operation modes in Scenario 1, we recall the conditions in Table II.

The last subfigure in Fig. 5 and Table IV describe the diagnosed operation modes and the corresponding detection times. We see that all the operation modes are identified. The time duration of the mode diagnosis includes two periods. For example, we consider the time period from 0.2000 s to 0.2132 s. First, from 0.2000 s, the system is still in normal operation mode even though a fault happens. From $0.2132 \mathrm{~s}$, an unknown operation mode is declared since the feature set, $\mathbb{B}(t)$, is not close to any class. Until $0.2226 \mathrm{~s}$, Mode 2 is declared, and thus, the detection time is $0.0226 \mathrm{~s}$. The obtained detection times (in Table IV) are short enough for
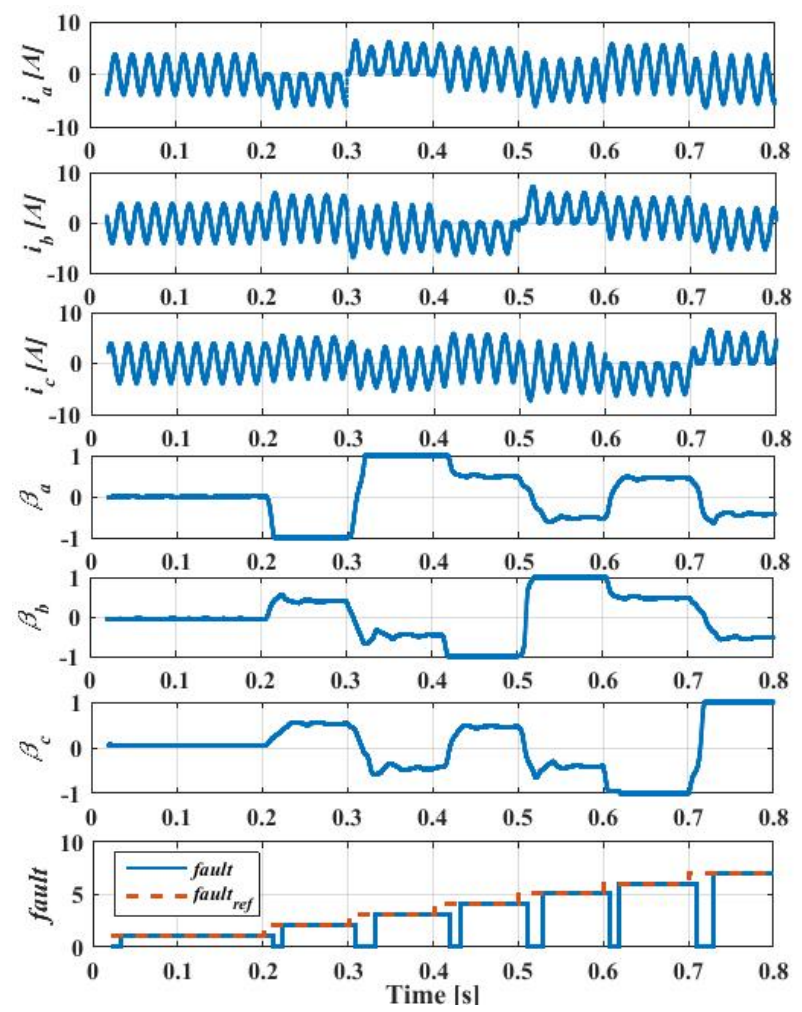

Fig. 5: Profiles of currents, of feature variables and of fault.

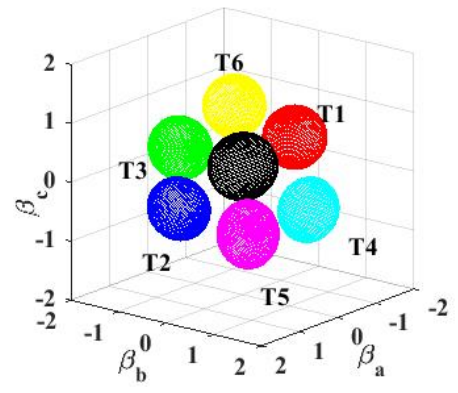

Fig. 6: Clustered classes in the feature space in Scenario 1.

turning off the system or reconfiguring the controller without causing damage to the inverter and motor.

B. Scenario 2: Experimental data

TABLE V: Settings and detection times in Scenario 2.

\begin{tabular}{|l|c|c|c|c|c|}
\hline Time [s] & 0.25 & 0.25 & 0.25 & 0.25 & 0.25 \\
\hline Fault & None & $T_{4}$ & $T_{3}$ & $T_{2}$ & $T_{1}$ \\
\hline fault $_{\text {ref }}$ & 1 & 5 & 4 & 3 & 2 \\
\hline Det. time [ms] & & 22.9 & 36.6 & 27.2 & 30.3 \\
\hline
\end{tabular}

Even though the paper focused on the 3-phase inverter, the procedure can be applied to 5-phase inverters with minimal changes as shown in this test case. The normal mode and 4 alternative single OC fault modes are implemented as given in Table V. Figure 7 illustrates the schematic diagram of the investigated 5-phase inverter associated to the 5-phase motor 
with the closed-loop regulator. The current profiles are shown in Fig. 8.

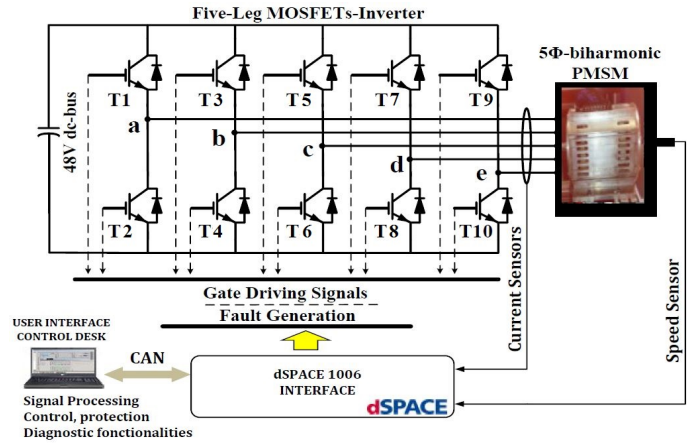

Fig. 7: Schematic diagram of the 5-phase inverter [1].
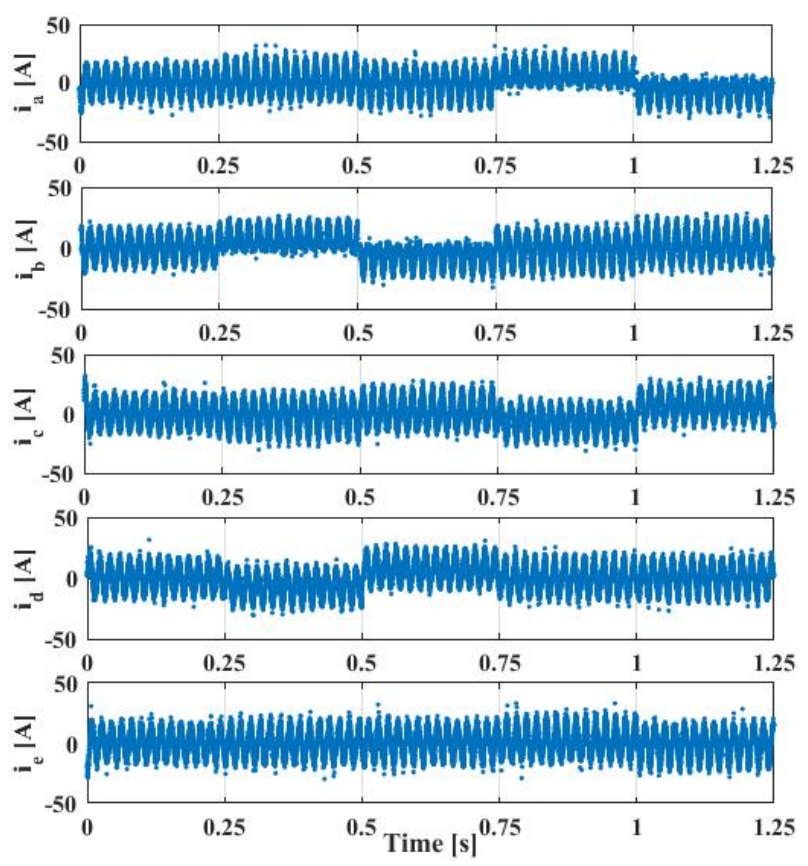

Fig. 8: Current profiles in Scenario 2.

\section{Conclusions}

This paper presented a hybrid diagnosis approach for the single open-circuit faults in inverters. The normalized DC current method is used to extract the features of different faulty operation modes based on the motor stator currents. In particular, the Auto-adaptive and Dynamical Clustering algorithm was employed for data clustering thanks to its online, adaptive and unsupervised learning capacities. Based on the obtained classes, we formulate the conditions for the labelling and fault detection and isolation. The approach was validated on simulation and experimental data. The short term future work concentrates on investigating the influence of the algorithm parameters on the detection time and making suggestions for choosing the ones yielding the smallest possible detection times. The algorithm robustness may be also evaluated with the load switching and/or intermittent fault. Moreover, the proposed approach will be compared with state of the art signal-based methods in the power electronics community.

\section{ACKNOWLEDGEMENT}

This work has been achieved within the framework of the CE2I project (Convertisseur d'Energie Intégré Intelligent). CE2I is co-financed by European Union with the financial support of European Regional Development Fund (ERDF), the French State and the French Region of Hauts-de-France. The authors would like also to thank Tiago Dos Santos Moraes, Eric Semail and Ngac Ky Nguyen from the L2EP laboratory in Lille (France) for providing the experimental data used in the second scenario.

\section{REFERENCES}

[1] M. Trabelsi, E. Semail, and N. K. Nguyen, "Experimental investigation of inverter open-circuit fault diagnosis for bi-harmonic five-phase permanent magnet drive," IEEE Journal of Emerging and Selected Topics in Power Electronic, vol. 6, no. 1, pp. 339 - 351, March 2018.

[2] B. Cai, Y. Zhao, H. Liu, and M. Xie, "A data-driven fault diagnosis methodology in three-phase inverters for PMSM drive systems," IEEE Transactions on Power Electronics, vol. 32, no. 7, pp. 5590 - 5600, July 2017.

[3] Z. Gao, C. Cecati, and S. X. Ding, "A survey of fault diagnosis and fault-tolerant techniques-part II: Fault diagnosis with knowledgebased and hybrid/active approaches," IEEE Transactions on Industrial Electronics, vol. 62, no. 6, pp. 3768 - 3774, June 2015.

[4] J. Poon, P. Jain, I. C. Konstantakopoulos, C. Spanos, S. K. Panda, and S. R. Sanders, "Model-based fault detection and identification for switching power converters," IEEE Transactions on Power Electronics, vol. 32, no. 2, pp. 1419 - 1430, February 2017.

[5] N. S. Ahmad, A. R. Abdullah, and N. Bahari, "Open and short circuit switches fault detection of voltage source inverter using spectrogram," Journal of International Conference on Electrical Machines and Systems, vol. 3, no. 2, pp. 190 - 199, 2014.

[6] J. O. Estima and A. J. M. Cardoso, "A new algorithm for real-time multiple open-circuit fault diagnosis in voltage-fed PWM motor drives by the reference current errors," IEEE Transactions on Industrial Electronics, vol. 60, no. 8, pp. 3496 - 3505, August 2013.

[7] D.-E. Kim and D.-C. Lee, "Fault diagnosis of three-phase PWM inverters using wavelet and SVM," Journal of Power Electronics, vol. 9, no. 3, pp. 377 - 385, May 2009.

[8] C. Delpha, D. Diallo, H. A. Samrout, and N. Moubayed, "Multiple incipient fault diagnosis in three-phase electrical systems using multivariate statistical signal processing," Engineering Applications of Artificial Intelligence, vol. 73, pp. 68 - 79, 2018.

[9] J. F. Martins, V. Fernao-Pires, and A. J. Pires, "Unsupervised neuralnetwork-based algorithm for an on-line diagnosis of three-phase induction motor stator fault," IEEE Transactions on Industrial Electronics, vol. 54, no. 1, pp. $259-264$, February 2007.

[10] M. A. Masrur, Z. Chen, and Y. Murphey, "Intelligent diagnosis of open and short circuit faults in electric drive inverters for real-time applications," IET Power Electronics, vol. 3, no. 2, pp. 279 - 291, March 2010.

[11] L. Meng, P. Wang, Z. Liu, R. Qiu, L. Wang, and C. Xu, "Safety assessment for electrical motor drive system based on SOM neural network," Mathematical Problems in Engineering, vol. 2016, 2016, 8 pages.

[12] M. Talha, F. Asghar, and S. H. Kim, "A Matlab and Simulink based three-phase inverter fault diagnosis method using three-dimensional features," International Journal of Fuzzy Logic and Intelligent Systems, vol. 16, no. 3, pp. 173 - 180, September 2016.

[13] S. Lecoeuche, C. Lurette, and S. Lalot, "New supervision architecture based on on-line modelling of non-stationary data," Neural Computing \& Applications, vol. 13, no. 4, pp. 323 - 338, December 2004.

[14] H. A. Boubacar, S. Lecoeuche, and S. Maouche, "Audyc neural network using a new gaussian densities merge mechanism," in Adaptive and Natural Computing Algorithms. Springer, 2005, pp. 155 - 158. 